\title{
Sleep, Immunity, and Its Impact on COVID Vaccination
}

\author{
Dhivya Narayanasamy ${ }^{1}$, Renuka Kandasamy²
}

\begin{abstract}
Coronaviruses are a large group of viruses that cause illness in humans and animals. The outbreak of coronavirus disease-2019 (COVID-19) was initially noticed in a seafood market in Wuhan, China, in mid-December 2019. A person who has had COVID-19 infection confirmed in the laboratory, regardless of clinical symptoms. The Pfizer-BioNTech COVID-19 vaccine, the Moderna COVID-19 vaccine, and the Janssen (Johnson and Johnson) COVID-19 vaccine have all been approved by the US Food and Drug Administration (FDA) for emergency use. The importance of getting enough good sleep for your immune system cannot be overstated. Sleep is essential for a healthy immune system, and as COVID-19 vaccines are distributed, it is critical that people get enough sleep to stay healthy, and to maintain optimal health, people must continue to prioritize their sleep. "Getting enough, high-quality sleep on a daily basis boosts the immune system and optimizes overall reaction to a vaccine." Normal sleep after vaccination boosts the immune response against an invading antigen, according to studies, and this immunity-boosting effect of sleep is clinically significant. "Getting a good night's sleep before and after immunization may be beneficial."
\end{abstract}

Keywords: COVID vaccination, Immunity, Sleep.

Pondicherry Journal of Nursing (2021): 10.5005/jp-journals-10084-13119

\section{INTRODUCTION}

Coronavirus disease-2019 (COVID-19) is the name of the disease that can be caused by the virus severe acute respiratory syndrome coronavirus 2 (SARS-CoV-2), a new form of coronavirus that was first discovered in late 2019 in Wuhan, China. SARS-CoV-2 is most easily transmitted through respiratory droplets when people are within $6 \mathrm{ft}$ of one another. The virus can also be spread through the air, in the form of aerosols that are produced when people talk or breathe and can linger in the air for a long time.

Airborne viral transmission can occur even when people are more than $6 \mathrm{ft}$ apart, and it is more likely in enclosed areas with poor ventilation.

\section{History}

Coronaviruses are a broad genus of viruses that infect both people and animals. In mid-December 2019, an epidemic of coronavirus sickness was discovered in a seafood market in Wuhan, China. On January 30, 2020, the World Health Organization (WHO) designated the epidemic a "Public Health Emergency of International Concern."

\section{COVID-19 CASE}

A person who has had COVID-19 infection confirmed in the laboratory, regardless of clinical symptoms.

\section{COVID-19 Symptoms}

- It varies a lot across people who have been infected with the virus. Fever, cough, difficulty breathing, loss of taste and/or smell, headache and/or body soreness, nausea, vomiting, diarrhea, nasal congestion, and runny nose are some of the symptoms that patients experience.

- COVID-19 can cause difficulty breathing; chest pain or pressure; confusion; inability to wake up or stay awake; and pale/discolored skin, lips, or nail beds in severe cases. Please get emergency medical assistance if you are experiencing these symptoms.
1,2Department of Medical Surgical Nursing, Kasturba Gandhi Nursing College, Puducherry, India

Corresponding Author: Dhivya Narayanasamy, Department of Medical Surgical Nursing, Kasturba Gandhi Nursing College, Puducherry, India, Phone: +918524045305, e-mail: ndhivya0710@ gmail.com

How to cite this article: Narayanasamy D, Kandasamy R. Sleep, Immunity, and Its Impact on COVID Vaccination. Pon J Nurs 2021;14(4):88-90.

Source of support: Nil

Conflict of interest: None

- People who have been infected but are asymptomatic or presymptomatic (not yet showing symptoms) have the potential to spread the virus to others. ${ }^{3}$

\section{Types of COVID-19 Vaccines}

The Pfizer-BioNTech COVID-19 vaccine, the Moderna COVID-19 vaccine, and the Janssen (Johnson and Johnson) COVID-19 vaccine have all been approved by the US Food and Drug Administration (FDA) for emergency use. Each of these vaccines is delivered by injecting it into the upper arm muscle. ${ }^{4}$

COVID-19 vaccinations have been proved to be safe in 10s of 1000 s of patients in numerous nations, and this safety record has been backed up by the fact that over 75 million doses have been delivered in the United States since December $2020 .^{5}$

\section{Prevaccination Precautions}

- Have known allergies.

- Have a fever.

- Have symptoms of COVID-19 or were recently exposed to an infected person.

- Are using a blood thinner.

- Have a bleeding disorder.

(O) The Author(s). 2021 Open Access This article is distributed under the terms of the Creative Commons Attribution 4.0 International License (https://creativecommons. org/licenses/by-nc/4.0/), which permits unrestricted use, distribution, and non-commercial reproduction in any medium, provided you give appropriate credit to the original author(s) and the source, provide a link to the Creative Commons license, and indicate if changes were made. The Creative Commons Public Domain Dedication waiver (http://creativecommons.org/publicdomain/zero/1.0/) applies to the data made available in this article, unless otherwise stated. 
- Are immunocompromised.

- Take medications that affect your immune system, talk to your doctor before getting vaccinated.

- Have already received a different COVID-19 vaccine.

- These circumstances do not preclude someone from receiving vaccinations, but discussing them with a doctor can help ensure that vaccines are administered safely. ${ }^{6}$

\section{Contraindications for Vaccination}

- People who have had an adverse reaction to a previous dose of the COVID-19 vaccine or to any of the vaccine's ingredients should not have the vaccination.

- Vaccines are not approved for use in children at this time. The Pfizer vaccination is approved for people aged 16 and above, while the Moderna and Janssen vaccinations are approved for persons aged 18 and above. ${ }^{7}$

\section{Debunking Myths of COVID-19}

- Despite the fact that major research studies have established that COVID-19 vaccinations are safe, certain falsehoods about them continue to circulate online. Knowing the truth can assist in dispelling such myths.

- The COVID-19 vaccine does not protect you from the virus: Only little bits of coronavirus materials are used in the vaccines, and these pieces are unable to spread the virus. In fact, by teaching your immune system to work together, you can improve your overall health.

- The COVID-19 vaccines have no effect on your deoxyribonucleic acid (DNA): None of the vaccines has the ability to penetrate the nucleus of your cells, which is where your DNA is found.

- Female fertility is unaffected by COVID-19 vaccines: No studies have found that these or any other vaccines have an influence on a woman's capacity to conceive. The immunizations are also thought to be safe for women who are already expecting a child.

\section{Sleep, Immunity, and COVID Vaccination}

The importance of getting enough good sleep for your immune system cannot be overstated. Although there is no research on sleep and the COVID-19 vaccines, investigations of other vaccines have revealed that sleep deprivation can diminish their effectiveness.

Sleep is essential for a healthy immune system, and as COVID-19 vaccines are distributed, it is critical that people get enough sleep to stay healthy.

"As COVID-19 vaccines are disseminated, it is critical that patients prioritize their sleep in order to maintain optimal health." "Getting enough, high-quality sleep on a regular basis boosts the immune system and optimizes the vaccine response." ${ }^{8}$

Sleep has an impact on a variety of immunological parameters, and getting enough sleep is linked to a lower risk of infection, a better infection outcome, and a better vaccine response. Sleep deprivation, on the contrary, has been demonstrated to damage the body's ability to protect itself. White blood cells (immune system cells) that go to damaged bodily areas are reduced. ${ }^{9}$

Patients who are sleep deprived (less than 7 hours of sleep per night) have a weaker immune system response to vaccines, especially the flu shot, and are 11 times more likely to stay vulnerable despite vaccination. Those who suffer from sleep disorders may be concerned about how the vaccine will affect their current medications or whether it may worsen their symptoms. "There is no indication that it is likely to worsen any sleep condition, and all the patients are enlightened."
Vaccinating patients with sleep apnea is especially crucial because COVID-19 is linked to poorer outcomes. The importance of sleep in enhancing innate and acquired immune responses cannot be overstated. Everyone, especially health professionals, should be aware of the immune-boosting benefits of sleep. Normal sleep after vaccination boosts the immune response against an invading antigen, according to studies, and this immunity-boosting effect of sleep is clinically significant. "Getting a good night's sleep before and after immunization may be beneficial."

\section{Nursing Care of COVID-19 Vaccine}

Nurses play a critical role in vaccine administration and organization, particularly during the COVID-19 pandemic.

Nurses help in this effort by educating vaccine recipients, ensuring public awareness, and physically administering immunizations. A nurse's function in the immunization process is defined as follows:

- Communication: Nurses provide communication and care to the public, which ultimately leads to personal and parental healthcare decisions. Nurses can educate patients about vaccine efficacy and safety by staying informed about the importance and procedure of required vaccinations. As a result, the most important function nurses play in the vaccination process is communication-and the subsequent public awareness.

- Administrative safety: Another challenge that nurses must overcome is ensuring the safe handling, storage, and administration of immunizations. In ensuring a safe immunization process, nurses must also take patients' health history and be aware of any allergies.

- Follow-up: Nurses are also in charge of all aspects of the immunization procedures once it has been administered.

\section{Role of Nurses before Vaccination}

Nurses should educate the people about the following steps:

- Do not take the vaccine while hungry. Before getting immunization, eat a healthy meal, including leafy greens, which are high in antioxidants and can aid to increase immunity.

- Stay hydrated by drinking plenty of water.

- Get a good, restful night's sleep. A good night's sleep has been scientifically demonstrated to help increase immunity.

- To avoid adverse effects, do not take any painkiller medications before getting the immunization.

- Be ready to receive any vaccine that is offered at the moment at the center. Keep in mind that all vaccines have been shown to be safe and effective.

- To make getting the injection on arm is easier, wear a sleeveless or short-sleeved blouse or dress.

- If you notice any COVID-like symptoms and need the doctor's approval, cancel the visit.

- Bring the identification you used to register. Wear a double mask and keep a safe distance from the center. Take note of the type of vaccine you received, since this will help you schedule an appointment for the second dosage.

\section{Nursing Care after Vaccination}

Here are some nursing care after vaccination:

- After the vaccination, sit for 30 minutes at the immunization center. The nurse should keep an eye on the patient for any 
unexpected reactions. If you feel dizzy, nauseated, vomiting, or have an allergic response, please report it.

- After receiving the vaccine, drink plenty of water to stay hydrated.

- Do not drink alcohol or smoke.

- Swing the vaccinated arm to reduce inflammation by applying a cool or moist towel to the region. Be prepared for some minor side effects as your body builds immune protection. These may go away in 1-2 days. The side effects are as follows:

- Pain and redness at the site of injection.

- Chills and mild fever.

- Headache.

- Fatigue and body ache.

- Joint and muscle pain.

- If you have any of the symptoms listed above, take paracetamol. If they persist for more than a week, see the doctor right once.

- Maintain a 14-day gap after taking the COVID-19 vaccine if you want to get another vaccine for a different medical problem. Consult with a doctor about any recent vaccinations that have been received.

- Download your immunization certificate, put the second dosage of the vaccine in your calendar, and make an appointment.

- You may suffer similar adverse effects following the second dosage of the vaccine as you had after the first. Call your doctor if the adverse effects last longer than 1-2 days.

- Remember that after 15 days, the antibodies developed after the second dosage of the vaccination reach a protective level. As a result, use caution and do not relax your preventive measures. If you have been using immunosuppressants like methotrexate, it is equally crucial to talk to your doctor about it. Following the COVID-19 immunization, doctors may put the drug on hold for two weeks.

\section{Conclusion}

Getting enough, high-quality sleep on a regular basis boosts the immune system and optimizes the vaccine response. And also, sleep is linked to a lower risk of infection and a better infection outcome. In addition, the FDA and the Centers for Disease Control and Prevention (CDC) are continuing a number of projects that monitor vaccine responses in order to discover reports of side effects immediately and engage with healthcare practitioners to ensure that immunization programs are safe.

\section{OrCID}

Renuka Kandasamy 난 htps://orcid.org/0000-0001-8898-4515

\section{References}

1. World Health Organization. WHO Director-General's opening remarks at the media briefing on COVID-19-11 March 2020. 2020. Available from: https://www.who.int/director-general/speeches/detail/whodirector-general-s-opening-remarks-at-the-media-briefing-oncovid-19-11-march-2020 [Retrieved March 5, 2021].

2. U.S. Food and Drug Administration. Emergency use authorization for vaccines explained. 2020. Available from: https://www.fda.gov/ vaccines-blood-biologics/vaccines/emergency-use-authorizationvaccines-explained [Retrieved March 5, 2021].

3. World Health Organization. Naming the coronavirus disease (COVID-19) and the virus that causes it. n.d. Available from: https://www.who.int/ emergencies/diseases/novel-coronavirus-2019/technical-guidance/ naming-the-coronavirus-disease-(covid-2019)-and-the-virus-thatcauses-it [Retrieved March 5, 2021].

4. National Center for Immunization and Respiratory Diseases (NCIRD), Division of Viral Diseases, CDC. How COVID-19 spreads. Centers for Disease Control and Prevention; 2020. Available from: https://www. cdc.gov/coronavirus/2019-ncov/prevent-getting-sick/how-covidspreads.html [Retrieved March 3, 2021].

5. U.S. Food and Drug Administration. Janssen COVID-19 vaccine. 2021. Available from: https://www.fda.gov/emergency-preparednessand-response/coronavirus-disease-2019-covid-19/janssen-covid-19vaccine [Retrieved March 3, 2021].

6. Chung YH, Beiss V, Fiering SN, Steinmetz NF. COVID-19 vaccine frontrunners and their nanotechnology design. ACS Nano 2020;14(10):12522-12537. DOI: 10.1021/acsnano.0c07197. Available from: https://pubmed.ncbi.nlm.nih.gov/33034449/.

7. U.S. Food and Drug Administration. Pfizer-BioNTech COVID-19 vaccine frequently asked questions. 2021. Available from: https:// www.fda.gov/emergency-preparedness-and-response/mcm-legalregulatory-and-policy-framework/pfizer-biontech-covid-19-vaccinefrequently-asked-questions [Retrieved March 3, 2021].

8. Bangaru S, Ozorowski G, Turner HL, Antanasijevic A, Huang D, Wang X, et al. Structural analysis of full-length SARS-CoV-2 spike protein from an advanced vaccine candidate. Science 2020;370(6520):10891094. DOI: 10.1126/science.abe1502. Available from: https:// pubmed.ncbi.nlm.nih.gov/33082295/.

9. National Center for Immunization and Respiratory Diseases (NCIRD), Division of Viral Diseases, CDC. SARS-CoV-2 variants. Centers for Disease Control and Prevention. 2020. Available from: https:// www.cdc.gov/coronavirus/2019-ncov/cases-updates/variantsurveillance/variant-info.html [Retrieved March 3, 2021]. 